Collection SFN 7 (2007) 173-197

(C) EDP Sciences, Les Ulis

DOI: $10.1051 /$ sfn:2007022

\title{
Disordered materials studied using neutron polarization analysis
}

\section{J. Ross Stewart ${ }^{1}$}

${ }^{1}$ Institut Laue-Langevin, 6 rue Jules Horowitz, 38042 Grenoble, France

\begin{abstract}
The technique of longitudinal polarization analysis on a multi-detector ("xyz"- polarization analysis) is presented. This technique allows the simultaneous and unambiguous determination of the nuclear, magnetic and nuclear spin-incoherent scattering functions as a function of both momentum and energy transfer. Several examples of neutron polarization analysis studies of disordered systems are given, illustrating the valuable information which can be retrieved from the analysis of neutron scattering between the Bragg peaks.
\end{abstract}

\section{INTRODUCTION}

The often ignored portions of neutron diffraction patterns of crystalline materials - i.e. those between the nuclear or magnetic Bragg peaks - provide valuable information about the degree of disorder, or more correctly, the degree of deviation from perfect long-range order, in the sample under investigation. This contribution to the total scattering is termed the diffuse scattering. There are a number of possible causes of nuclear diffuse scattering, examples of which include: the presence of nuclear isotopes, nuclear spin-incoherent scattering, random or non-random solid solution materials (e.g. binary alloys), interstitial defects, vacancies and internal strain. If the material under investigation is magnetic, or contains some magnetic species, then any disordered (paramagnetic) moment will result in diffuse scattering. Long range ordered magnetic materials with magnetic defects also display diffuse scattering. However, magnetic diffuse scattering is often weak, and usually obscured by the nuclear diffuse scattering, as well as thermal diffuse scattering caused by phonons and incoherent thermal excitations in the sample. Using polarized neutrons, one may simultaneously and unambiguously extract the nuclear and magnetic cross-sections, on a multi-detector spectrometer - in a "flat-cone" type geometry appropriate for diffuse scattering studies of powders and single crystals.

This article will concentrate on the use of polarized neutrons in the extraction of the nuclear and magnetic diffuse scattering contributions of disordered materials. The use of polarization analysis in order to separate collective and single particle excitations in non-magnetic materials will also be discussed.

\section{DIFFUSE SCATTERING INSTRUMENTATION}

\subsection{General requirements}

In the design of a neutron spectrometer dedicated to the study of diffuse scattering from crystalline materials, there are several important design considerations.

(a) The instrument should operate at long wavelengths (cold neutrons). Diffuse scattering experiments should ideally be conducted above the Bragg cut-off wavelength, i.e. where the incident neutron wavelength $\lambda>2 d_{\max }$, where $d_{\max }$ is the d-spacing of the first allowed Bragg reflection. This avoids the possibility of multiple Bragg scattering which would appear on top of the diffuse scattering, and render the data difficult to analyse.

(b) Due to the fact that diffuse scattering contributions vary smoothly with wavevector transfer Q, (no sharp features) Q-resolution should be relaxed in favour of high neutron flux. 
(c) Since diffuse scattering is generally weak, and rather flat, the instrumental background needs to be kept as low as possible. This normally entails secondary collimation before the detectors in order to ensure that counts are not recorded which come from scattering from the sample environment.

(d) The instrument should have the ability to differentiate truly elastic scattering from quasi-elastic or inelastic contributions. Such contributions to the diffuse scattering are termed thermal diffuse scattering (TDS). This requires either an analyser to determine the final neutron energy (i.e. a three-axis spectrometer), or time-of-flight analysis of a pulsed or otherwise time modulated neutron beam.

(e) The instrument should be equipped with the ability to perform neutron polarization analysis, so that the nuclear coherent, nuclear spin-incoherent and magnetic contributions to the diffuse scattering can be unambiguously determined.

(f) Since it is also a distinct advantage to be able to measure as wide an area of reciprocal space as possible, it is desirable that the instrument be equipped with a wide angle multi-detector in a "flat-cone" type geometry - enabling single-shot powder studies, and omega-scan type maps of planes in reciprocal space for single crystal samples.

To date there are three instruments in the world which fulfill the above criteria. They are : DNS at the FRJ-II reactor in Jülich, Germany [1], LONGPOL at the HIFAR reactor in Lucas Heights, Australia [2], and D7 at the Institut Laue-Langevin (ILL) in Grenoble, France [3].

\subsection{The Diffuse Scattering Spectrometer D7}

D7 was one of the first instruments built in the cold neutron guide hall at the ILL. It was originally a flat-cone multi-detector spectrometer, with a single disc chopper providing very relaxed resolution energy analysis so that TDS could be separated out [4]. From the late 70s onwards, it had the option

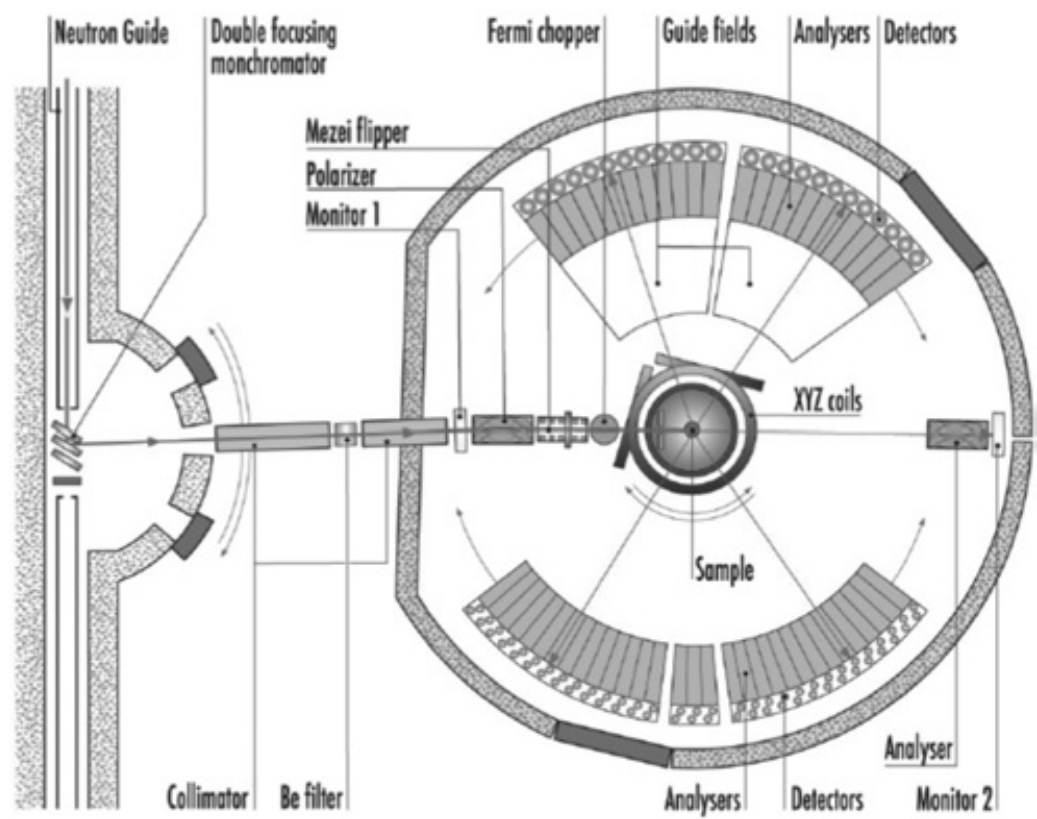

Figure 1. The D7 diffuse scattering spectrometer at the ILL [7]. 
of polarizing the incident beam, enabling studies of ferromagnetic materials (see section 7.1). In the early 80s, D7 was equipped with supermirror analyser detector banks, allowing uniaxial and 3-directional longitudinal polarization analysis [5] (the so-called “xyz"-method). The current D7 instrument has been rebuilt as part of the ILL Millennium Project, and is shown in Figure (1). It has a doubly focusing pyrolytic graphite monochromator, with available incident wavelengths of $3.1 \AA$, $4.8 \AA$ and $5.9 \AA$. The neutron beam is spin-polarized and spin-analysed by $m=3$ supermirror Schärpf benders [6]. The complete spectrometer contains over $260 \mathrm{~m}^{2}$ of supermirror (enough to cover a tennis court). The polarized neutron flux is $3 \times 10^{6} \mathrm{n} \mathrm{cm}^{-2} \mathrm{~s}^{-1}$. A Fermi-chopper of relaxed resolution enables time-of-flight energy analysis. D7 complies with all the desired criteria for diffuse scattering instrumentation listed above, with the exception that the incoming divergence available is limited by the acceptance of the $m=1$ $\mathrm{Ni}$-coated neutron guide. This disadvantage is compensated for by the fact that the neutron background in the ILL guide hall is much less than would be the case if D7 were placed on a standard neutron beam tube near to the reactor.

\section{LONGITUDINAL OR UNIAXIAL NEUTRON POLARIZATION ANALYSIS}

\subsection{Introduction}

In order to achieve separation of magnetic and nuclear diffuse scattering cross-sections in a neutron scattering experiment, analysis of the spin of the scattered neutrons is required. The equations for the spin-dependent neutron scattering amplitudes may be deduced from the equation for the elastic neutron scattering cross-section (which is straightforwardly generalized to include the inelastic case)

$$
\frac{d \sigma}{d \Omega}=\left(\frac{m_{n}}{2 \pi \hbar^{2}}\right)^{2}\left|\left\langle\overrightarrow{k^{\prime}} \overrightarrow{s^{\prime}}|V| \vec{k} \vec{S}\right\rangle\right|^{2}
$$

If we impose a magnetic field onto our scattering experiment, the neutron spin (spin-1/2) is quantized into its two Zeeman states, either parallel or anti-parallel to the quantization axis given by the external magnetic field. All neutron polarization analysis experiments carried out under these conditions are termed longitudinal polarization analysis (LPA) experiments, since it is only the scalar component of the neutron spin along the field direction which is measurable. All transverse components will rapidly precess around the field direction and not be measurable in the experiment. In an LPA experiment we donate the two spin-states by the spinors

$$
\begin{aligned}
& |+\rangle=\left(\begin{array}{l}
1 \\
0
\end{array}\right) \\
& |-\rangle=\left(\begin{array}{l}
0 \\
1
\end{array}\right)
\end{aligned}
$$

\subsection{The Moon-Riste-Koehler Equations}

In general a bound state is formed between the nucleus and the neutron during scattering with either spins anti-parallel (spin-singlet) or spins parallel (spin-triplet). The scattering lengths for these situations are different and are termed $b_{-}$and $b_{+}$(these - and + scattering lengths are given explicitly in the neutron scattering tables compiled by Rauch and Waschkowski [8]). We define the nuclear scattering length operator [9]

$$
\hat{\mathbf{b}}=A+B \sigma \cdot \vec{I}
$$

where $\sigma$ is the Pauli spin operator and

$$
A=\frac{(I+1) b_{+}+I b_{-}}{2 I+1}, \text { and } B=\frac{b_{+}-b_{-}}{2 I+1}
$$


The nuclear scattering potential is then given by the Fermi-pseudopotential

$$
V=\frac{2 \pi \hbar^{2}}{m_{n}} \widehat{\mathbf{b}}
$$

and the spin-dependent scattering amplitudes are given by

$$
\left\langle\overrightarrow{S^{\prime}}|\widehat{\mathbf{b}}| \vec{S}\right\rangle=\left\{\begin{aligned}
A+B I_{z} & |+\rangle \rightarrow|+\rangle \\
A-B I_{z} & |-\rangle \rightarrow|-\rangle \\
B\left(I_{x}-i I_{y}\right) & |+\rangle \rightarrow|-\rangle \\
B\left(I_{x}+i I_{y}\right) & |-\rangle \rightarrow|+\rangle
\end{aligned}\right\} \text { spin flip }
$$

Since the nuclear spins are (normally) random, we can state that $\left\langle I_{z}\right\rangle=\left\langle I_{x}\right\rangle=\left\langle I_{y}\right\rangle=0$, and therefore the coherent scattering amplitude is given by

$$
\bar{b}=A
$$

The magnetic scattering potential $V_{m}$ is given by

$$
V_{m}(\vec{Q})=-\frac{\gamma_{n} r_{0}}{2 \mu_{B}} \sigma \cdot \mathbf{M}_{\perp}(\vec{Q})=-\frac{\gamma_{n} r_{0}}{2 \mu_{B}} \sum_{\zeta} \sigma_{\zeta} M_{\perp \zeta}(\vec{Q})
$$

where $\zeta=x, y, z$. Here, $\mathbf{M}_{\perp}(\vec{Q})$ represents the so-called transverse magnetization operator which is the Fourier transform of the magnetization of the sample perpendicular to the scattering vector $\vec{Q}$ - i.e. the neutron sensitive magnetization [10]. We therefore arrive at the magnetic scattering amplitudes

$$
\left\langle\overrightarrow{S^{\prime}}\left|V_{m}(\vec{Q})\right| \vec{S}\right\rangle=-\frac{\gamma_{n} r_{0}}{2 \mu_{B}}\left\{\begin{array}{cc}
M_{\perp z}(\vec{Q}) & |+\rangle \rightarrow|+\rangle \\
-M_{\perp z}(\vec{Q}) & |-\rangle \rightarrow|-\rangle
\end{array}\right\} \text { non spin flip }
$$

This equation illustrates one of the main rules of thumb which is very useful to remember and is the basis of all magnetic uniaxial neutron polarization analysis measurements. Remembering that the neutron polarization is aligned along the $z$ - direction we can state:

The non-spin-flip scattering is sensitive only to those components of the sample magnetization which are parallel to the neutron spin.

The spin-flip scattering is sensitive only to those components of the sample magnetization which are perpendicular to the neutron spin.

Bringing together the nuclear and magnetic scattering amplitudes for a uniaxial polarized beam, we arrive at the so-called Moon-Riste-Koehler equations [11] for the spin dependent scattering amplitudes

$$
\begin{aligned}
U^{|+\rangle \rightarrow|+\rangle} & =\widehat{b}-p M_{\perp z}+B I_{z} \\
U^{|-\rangle \rightarrow|-\rangle} & =\widehat{b}+p M_{\perp z}-B I_{z} \\
U^{|+\rangle \rightarrow|-\rangle} & =p \prime\left(M_{\perp x}-i M_{\perp y}\right)+B\left(I_{z}-i I_{y}\right) \\
U^{|-\rangle \rightarrow|+\rangle} & =p \prime\left(M_{\perp x}+i M_{\perp y}\right)+B\left(I_{z}+i I_{y}\right)
\end{aligned}
$$

where $p$ is the magnetic prefactor $-\frac{\gamma_{n} r_{0}}{2 \mu_{B}}$. 


\section{INCOHERENT SCATTERING}

\subsection{Nuclear Incoherent Scattering}

The most basic form of diffuse scattering is incoherent scattering. Whereas the coherent scattering from a crystal depends on spatial and temporal correlations between the same nucleus or different nuclei at different times, the incoherent scattering arises from self-correlations of the same nucleus at different times. The neutron scattering cross-section (integrated over energy) is

$$
\frac{d \sigma}{d \Omega}=\sum_{i, j} b_{i} b_{j} \exp \left[i \vec{Q} \cdot\left(\vec{R}_{i}-\vec{R}_{j}\right)\right] \approx \sum_{i, j} \overline{b_{i} b_{j}} \exp \left[i \vec{Q} \cdot\left(\vec{R}_{i}-\vec{R}_{j}\right)\right]
$$

where $b_{i}$ and $b_{j}$ are the scattering lengths of the $i$ th and $j$ th nuclei, and assuming that the sum is taken over a sufficiently large number of scatterers (usually well satisfied!). Now, if we make that assumption that there are no correlations between the atomic species, we can state that,

$$
\overline{b_{i} b_{j}}=\left\{\begin{array}{cl}
(\bar{b})^{2} & \text { for } \quad i \neq j \\
\bar{b}^{2} & \text { for } \quad i=j
\end{array}\right.
$$

Therefore we can split the equation for the energy integrated neutron scattering cross-section, eq.4.1 into self and distinct parts as follows.

$$
\begin{aligned}
\frac{d \sigma}{d \Omega} & =\sum_{i, j, i \neq j}(\bar{b})^{2} \exp \left[i \vec{Q} \cdot\left(\vec{R}_{i}-\vec{R}_{j}\right)\right]+\sum_{i} \overline{b^{2}} \exp \left[i \vec{Q} \cdot\left(\vec{R}_{i}-\vec{R}_{i}\right)\right] \\
& =\sum_{i, j}(\bar{b})^{2} \exp \left[i \vec{Q} \cdot\left(\vec{R}_{i}-\vec{R}_{j}\right)\right]+\left[\overline{b^{2}}-(\bar{b})^{2}\right]
\end{aligned}
$$

The first term - the coherent scattering - contains the scattering cross-section that would be obtained if all the scattering centres had the same average scattering length $\widehat{b}$. The second term which represents the incoherent scattering is the additional scattering caused by randomly distributed deviations from the mean scattering length.

\subsection{Spin Dependent Nuclear Incoherent Scattering}

From eq. 4.3 the incoherent scattering cross-section is given by

$$
\left(\frac{d \sigma}{d \Omega}\right)_{i n c}=\left[\overline{b^{2}}-(\bar{b})^{2}\right]
$$

Now, applying this to the $|+\rangle \rightarrow \mid+$ ngle transition in eq. 3.7 we find,

$$
\begin{aligned}
\left(\frac{d \sigma}{d \Omega}\right)_{i n c} & =\overline{\left(\bar{b}+B I_{z}\right)^{2}}-(\bar{b})^{2} \\
& =\overline{(\bar{b})^{2}}+\overline{B^{2} I_{z}^{2}}+2 \overline{\bar{b} B I_{z}}-(\bar{b})^{2}
\end{aligned}
$$

For a randomly oriented distribution of nuclei, the expectation value of the nuclear spin operator $\mathbf{I}$ is given by,

$$
\langle\mathbf{I}\rangle=\sqrt{I(I+1)}=\sqrt{I_{x}^{2}+I_{y}^{2}+I_{z}^{2}}
$$


Since the distribution of spin components is isotropic, we can write

$$
I_{x}^{2}=I_{y}^{2}=I_{z}^{2}=\frac{1}{3} I(I+1)
$$

Therefore the expression for the spin-dependent nuclear incoherent scattering in the $|+\rangle \rightarrow|+\rangle$ neut1ron polarization channel is

$$
\left(\frac{d \sigma}{d \Omega}\right)_{i n c}=\overline{b^{2}}-(\bar{b})^{2}+\frac{1}{3} B^{2} I(I+1)
$$

The other spin channels are dealt with in a similar way. The first two terms in eq. 4.8 make up the nuclear isotope incoherent scattering, and the last term is the nuclear spin-incoherent scattering. We may now rewrite the Moon-Riste-Koehler equations 3.10 as follows.

$$
\begin{aligned}
& U^{|+\rangle \rightarrow|+\rangle}=\widehat{b}-p M_{\perp z}+b_{i i}+\frac{1}{3} b_{s i} \\
& U^{|-\rangle \rightarrow|-\rangle}=\widehat{b}+p M_{\perp z}+b_{i i}+\frac{1}{3} b_{s i} \\
& U^{|+\rangle \rightarrow|-\rangle}=p \prime\left(M_{\perp x}-i M_{\perp y}\right)+\frac{2}{3} b_{s i} \\
& U^{|-\rangle \rightarrow|+\rangle}=p \prime\left(M_{\perp x}+i M_{\perp y}\right)+\frac{2}{3} b_{s i}
\end{aligned}
$$

where $b_{i i}=\sqrt{\overline{b^{2}}-(\bar{b})^{2}}$ is the isotope incoherent scattering length and $b_{s i}=\sqrt{\frac{1}{3} B^{2} I(I+1)}$ is the spin-incoherent scattering length. The incoherent scattering lengths normally quoted in scattering length tables (e.g. Sears [12] or Rauch [8]) are the addition of these two scattering lengths. The Moon-RisteKoehler equations now immediately suggest a method of separating the nuclear spin-incoherent scattering from the nuclear coherent and isotope incoherent scattering in the absence of magnetic scattering. Subtraction of half the spin flip scattering from the non spin flip scattering, leaves only the coherent scattering. This is particularly useful in the case of protonated polymer or biological samples where the presence of massive spin-incoherent scattering from hydrogen can hinder the determination of the coherent structure factors. Separation of spin-incoherent from coherent scattering also allows the separation of incoherent one particle phonon excitations from coherent co-operative excitations. Examples of polarization analysis studies of non-magnetic incoherent scatterers will be presented in section 6.2.

\subsection{Incoherent Magnetic Scattering - Paramagnetism}

The details of the magnetic scattering terms in the Moon-Riste-Koehler equations will in general depend on the direction of the neutron polarization with respect to the scattering vector, and also on the nature of the orientation of the magnetic moments. On diffuse scattering instruments with "xyz"- polarization analysis it is a simple matter to reverse the neutron spin direction in the primary beam using a spinflipper device - such as a Mezei coil [13] or a current sheet [14]. However, due to the large solid angle of the multi-detector, it is difficult to spin-turn the scattered neutrons. The xyz polarization analysis technique therefore makes use only of two out of four of the scattering channels, with the final spin direction fixed. Assuming that the final spin direction is $|+\rangle$, we find the magnetic only cross-sections by squaring the magnetic part of the Moon-Riste-Koehler equations.

$$
\begin{aligned}
\left(\frac{d \sigma}{d \Omega}\right)_{n s f} & =\left(\frac{\gamma_{n} r_{0}}{2 \mu_{B}}\right)^{2}\left\langle M_{\perp z}^{*} M_{\perp z}\right\rangle \\
\left(\frac{d \sigma}{d \Omega}\right)_{s f} & =\left(\frac{\gamma_{n} r_{0}}{2 \mu_{B}}\right)^{2}\left\langle\left(M_{\perp x}+i M_{\perp y}\right)^{*}+\left(M_{\perp x}+i M_{\perp y}\right)\right\rangle \\
& =\left(\frac{\gamma_{n} r_{0}}{2 \mu_{B}}\right)^{2}\left\langle M_{\perp x}^{*} M_{\perp x}+M_{\perp y}^{*} M_{\perp y}\right\rangle
\end{aligned}
$$


where the subscripts $n s f$ and $s f$ denote the non spin flip and spin flip transitions respectively. It can be shown [15] that in the case of a paramagnet the magnetic scattering cross-sections become

$$
\begin{aligned}
\left(\frac{d \sigma}{d \Omega}\right)_{n s f} & =\frac{1}{3}\left(\frac{\gamma_{n} r_{0}}{2}\right)^{2} g_{S}^{2} f^{2}(\vec{Q}) S(S+1)\left[1-(\widehat{P} \cdot \widehat{Q})^{2}\right] \\
\left(\frac{d \sigma}{d \Omega}\right)_{s f} & =\frac{1}{3}\left(\frac{\gamma_{n} r_{0}}{2}\right)^{2} g_{S}^{2} f^{2}(\vec{Q}) S(S+1)\left[1+(\widehat{P} \cdot \widehat{Q})^{2}\right]
\end{aligned}
$$

We can immediately see that setting the direction of the polarization along the scattering vector renders all of the magnetic scattering in the spin-flip cross-section. The magnetic cross-section can therefore be isolated from the nuclear and the spin-incoherent scattering by measuring the difference between the spin-flip cross-sections measured with $\vec{P} \| \vec{Q}$ and $\vec{P} \perp \vec{Q}$, since

$$
\begin{array}{ll}
\widehat{P} \cdot \widehat{Q}=1: & \left(\frac{d \sigma}{d \Omega}\right)_{s f}=\frac{2}{3}\left(\frac{d \sigma}{d \Omega}\right)_{m a g}+\frac{2}{3}\left(\frac{d \sigma}{d \Omega}\right)_{s i} \\
\widehat{P} \cdot \widehat{Q}=0: & \left(\frac{d \sigma}{d \Omega}\right)_{s f}=\frac{1}{3}\left(\frac{d \sigma}{d \Omega}\right)_{m a g}+\frac{2}{3}\left(\frac{d \sigma}{d \Omega}\right)_{s i}
\end{array}
$$

Separation of magnetic scattering from other contributions using this technique is known as the parallel-perpendicular $(\|-\perp)$ method. It can be shown that this method also may be applied to antiferromagnetically ordered samples [5]. However the use of the $\|-\perp$ method is limited to measurements using a single detector so that the $\widehat{P} \cdot \widehat{Q}=1$ condition may be satisfied. The $\|-\perp$ technique was in use for several years on the D5 diffractometer at the ILL, and was used in studies of the diffuse magnetic scattering in materials such as $\mathrm{MnSi}[16]$ and $\mathrm{Fe}_{2} \mathrm{P}$ [17].

\section{5 “XYZ"- POLARIZATION ANALYSIS}

The technique of "xyz" or 3-directional neutron polarization analysis [5] provides full and unambiguous separation of the magnetic, nuclear and spin-incoherent cross-sections on a multi-detector neutron spectrometer. As such it is a technique well suited to neutron instruments which need to collect data over as wide a range of scattering angles as possible - i.e. diffuse scattering spectrometers and direct geometry time-of-flight inelastic spectrometers. It is a technique which is becoming especially relevant to instrumentation at spallation neutron sources where large solid-angle detector banks are frequently employed. "xyz"- polarization analysis has been used for over 25 years on D7 at the ILL.

\subsection{Elastic scattering}

If the geometry of the neutron spectrometer is chosen such that we have a planar multi-detector in the $x-y$ plane, the unit scattering vector is given by

$$
\widehat{Q}=\left(\begin{array}{c}
\cos \alpha \\
\sin \alpha \\
0
\end{array}\right)
$$

where the so-called "Schärpf angle" $\alpha$ is the angle between the scattering vector and the $x$-axis as depicted in Figure 2.

Substituting this scattering vector into the expression for the magnetic spin-dependent scattering given by Eq. 4.12 and 4.13, (and including the nuclear isotope incoherent and spin-incoherent terms from the Moon-Riste-Koehler equations 4.9) we arrive at the spin-dependent scattering cross-sections for an "xyz"- polarization analysis measurement, 


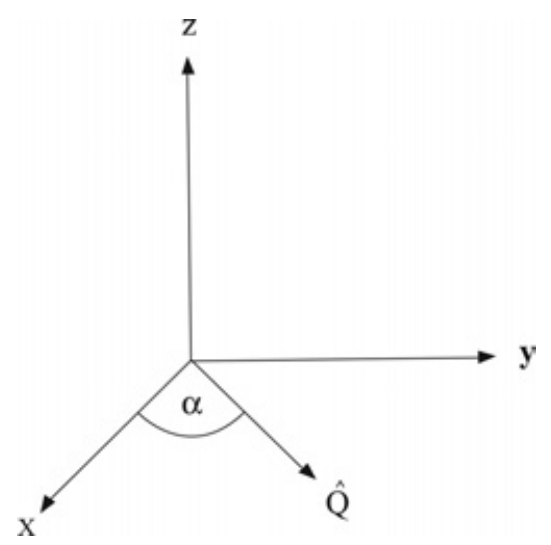

Figure 2. The geometry of an "xyz"- polarization analysis experiment. The incident polarization is alternately in the z-, $\mathrm{x}$ - and $\mathrm{y}$ - directions. The so-called Schärpf angle, $\alpha$, is the angle between the scattering vector and the arbitrarily defined $\mathrm{x}$ - direction.

$$
\begin{aligned}
\left(\frac{d \sigma}{d \Omega}\right)_{x}^{n s f} & =\frac{1}{2} \sin ^{2} \alpha\left(\frac{d \sigma}{d \Omega}\right)_{m a g}+\frac{1}{3}\left(\frac{d \sigma}{d \Omega}\right)_{s i}+\left(\frac{d \sigma}{d \Omega}\right)_{n u c+i i} \\
\left(\frac{d \sigma}{d \Omega}\right)_{x}^{s f} & =\frac{1}{2}\left(\cos ^{2} \alpha+1\right)\left(\frac{d \sigma}{d \Omega}\right)_{m a g}+\frac{2}{3}\left(\frac{d \sigma}{d \Omega}\right)_{s i} \\
\left(\frac{d \sigma}{d \Omega}\right)_{y}^{n s f} & =\frac{1}{2} \cos ^{2} \alpha\left(\frac{d \sigma}{d \Omega}\right)_{m a g}+\frac{1}{3}\left(\frac{d \sigma}{d \Omega}\right)_{s i}+\left(\frac{d \sigma}{d \Omega}\right)_{n u c+i i} \\
\left(\frac{d \sigma}{d \Omega}\right)_{s f}^{y} & =\frac{1}{2}\left(\sin ^{2} \alpha+1\right)\left(\frac{d \sigma}{d \Omega}\right)_{m a g}+\frac{2}{3}\left(\frac{d \sigma}{d \Omega}\right)_{s i} \\
\left(\frac{d \sigma}{d \Omega}\right)_{z s f}^{n s} & =\frac{1}{2}\left(\frac{d \sigma}{d \Omega}\right)_{\text {mag }}+\frac{1}{3}\left(\frac{d \sigma}{d \Omega}\right)_{s i}+\left(\frac{d \sigma}{d \Omega}\right)_{n u c+i i} \\
\left(\frac{d \sigma}{d \Omega}\right)_{z} & =\frac{1}{2}\left(\frac{d \sigma}{d \Omega}\right)_{\text {mag }}+\frac{2}{3}\left(\frac{d \sigma}{d \Omega}\right)_{s i}
\end{aligned}
$$

where the $x, y$ and $z$ subscripts refer to the direction of the incident polarization, and the $n s f$ and sf subscripts refer to the non-spin-flip and the spin-flip cross-sections respectively. The nuclear, spinincoherent and magnetic scattering cross-sections are then found via linear combinations of the "xyz"equations. The magnetic cross-section may be independently calculated in two ways,

$$
\left.\begin{array}{l}
\left(\frac{d \sigma}{d \Omega}\right)_{m a g}=2\left[\left(\frac{d \sigma}{d \Omega}\right)_{s f}^{x}+\left(\frac{d \sigma}{d \Omega}\right)_{s f}^{y}-2\left(\frac{d \sigma}{d \Omega}\right)_{s f}^{z}\right] \\
\left(\frac{d \sigma}{d \Omega}\right)_{m a g}=2\left[2\left(\frac{d \sigma}{d \Omega}\right)_{n s f}^{z}-\left(\frac{d \sigma}{d \Omega}\right)_{n s f}^{x}-\left(\frac{d \sigma}{d \Omega}\right)_{n s f}^{y}\right]
\end{array}\right\}=\frac{2}{3}\left(\frac{\gamma_{n} r_{0}}{2}\right)^{2} g_{S}^{2} f^{2}(\vec{Q}) S(S+1)
$$

For the nuclear and the spin-incoherent cross-sections we have

$$
\begin{aligned}
& \left(\frac{d \sigma}{d \Omega}\right)_{n u c+i i}=\frac{1}{6}\left[2\left(\frac{d \sigma}{d \Omega}\right)_{T n s f}-\left(\frac{d \sigma}{d \Omega}\right)_{T s f}\right]=b^{2} S(\vec{Q})+\overline{b^{2}}-(\bar{b})^{2} \\
& \left(\frac{d \sigma}{d \Omega}\right)_{s i}=\frac{1}{2}\left(\frac{d \sigma}{d \Omega}\right)_{T s f}-\left(\frac{d \sigma}{d \Omega}\right)_{m a g}=B^{2} I(I+1)
\end{aligned}
$$


where the subscripts Tnsf and Tsf refer to the total non-spin-flip and total spin-flip cross-sections. In the absence of magnetic scattering, one need only measure the $\mathrm{z}$-components of the spin-dependent cross-section in order to separate nuclear coherent and spin-incoherent scattering.

\subsection{Limitations of "xyz"- polarization analysis}

It should be reiterated here that the "xyz" equations apply only to magnetic systems with collinear magnetization and a randomly oriented moment direction - i.e. a paramagnetic or disordered static system or an antiferromagnetic polycrystalline system in zero external field. In antiferromagnetically ordered single crystals, there will in general be a strong correlation between the $x$ and $y$ components of the sample magnetization $\vec{M}$ - i.e. the components of $\vec{M}$ in the scattering plane. Therefore, in order to separate the magnetic cross-section in an ordered antiferromagnetic single crystal, either the angle between $\vec{M}$ and $\vec{Q}$ or the magnetic structure factor of the sample must be known in advance. In practice, if neither of these quantities are known, then application of the "xyz" equations will result in a possible change of sign of the magnetic intensity, depending on the moment direction [18]. In the case of non-collinear systems, such as helical magnets, cross-terms appear in the equations for the polarization dependent cross-section and the "xyz" equations break down.

It should also be stressed that "xyz" polarization analysis is an extension of unixial polarization analysis, and as such, the scattered polarization is not measured, but rather the longitudinal component of polarization along the magnetic guide field direction. This implies that any non-zero magnetization over long length scales will result in a reduction of this longitudinal component due to Larmor precession of the neutrons around the sample magnetization direction. Therefore, "xyz"-polarization analysis cannot be applied to the study of ferromagnets or even ferromagnetically correlated paramagnets with medium range correlations, since ferromagnetic domains and demagnetisation fields will depolarize the scattered neutrons. However, uniaxial polarization analysis of single domain ferromagnets (in a saturating magnetic field) can be performed in the direction of the sample magnetisation. This is particularly useful in so-called canted ferromagnetic systems or asperomagnetic systems. If the moment direction is locally canted away from the direction of the macroscopic sample magnetization, then spin-flip scattering from the transverse magnetization components will occur. The presence of spin-flip scattering is therefore a direct indication of moment canting. As an example of this sort of measurement, see the study of FeZr amorphous metals by Wildes and co-workers [19].

\subsection{Inelastic Scattering}

Analysis of the neutron polarization can also provide information on the inelasticity of the scattering process. The reason for this is that, in general, an inelastic scattering process will rotate the scattering vector $\vec{Q}$ around the arbitrary $x$ - axis, causing a change in the Schärpf angle $\alpha$. To demonstrate this we start by returning to the equations for paramagnetic scattering by a polarized beam, eq. 4.12 and 4.13. We may define the scalar polarization of the scattered beam as,

$$
P^{\prime}=\frac{\left(\frac{d \sigma}{d \Omega}\right)_{n s f}-\left(\frac{d \sigma}{d \Omega}\right)_{s f}}{\left(\frac{d \sigma}{d \Omega}\right)_{n s f}+\left(\frac{d \sigma}{d \Omega}\right)_{s f}}
$$

So substituting Eqs. 4.12 and 4.13 we get

$$
\begin{aligned}
P^{\prime} & =\left[\frac{\left(1-(\widehat{P} \cdot \widehat{Q})^{2}\right)-\left(1+(\widehat{P} \cdot \widehat{Q})^{2}\right)}{\left(1-(\widehat{P} \cdot \widehat{Q})^{2}\right)+\left(1+(\widehat{P} \cdot \widehat{Q})^{2}\right)}\right] \\
\Rightarrow \quad P^{\prime} & =-(\widehat{P} \cdot \widehat{Q})^{2}
\end{aligned}
$$




$$
\Rightarrow \quad \overrightarrow{P^{\prime}}=-(\widehat{P} \cdot \widehat{Q})^{2} \vec{P}
$$

Now, assuming "xyz"- polarization analysis geometry with $\vec{Q}$ given by eq. 5.1 we can write down the three components of the scattered polarization as

$$
\begin{aligned}
P_{x}^{\prime} & =-P_{x} \cos ^{2} \alpha \\
P_{y}^{\prime} & =-P_{y} \sin ^{2} \alpha \\
P_{z}^{\prime} & =0
\end{aligned}
$$

i.e. the direction of the scattered polarization from a paramagnetic material is the along the scattering vector. This direction of the scattered polarization is determined straightforwardly using "xyz"- polarization analysis using

$$
\tan ^{2} \alpha=\frac{\left(\frac{d \sigma}{d \Omega}\right)_{s f}^{y}-\left(\frac{d \sigma}{d \Omega}\right)_{s f}^{z}}{\left(\frac{d \sigma}{d \Omega}\right)_{s f}^{x}-\left(\frac{d \sigma}{d \Omega}\right)_{s f}^{z}}
$$

This then immediately shows whether or not the scattering vector has the same direction as that that would be expected for purely elastic scattering. "xyz"- polarization analysis therefore provides a method of inelastic neutron scattering without recourse to either time-of-flight analysis or crystal analysers. This method was used by Mezei and Murani [20], and more recently by Ehlers and co-workers [21].

\section{NUCLEAR DIFFUSE SCATTERING}

In this section, two types of nuclear diffuse scattering experiment will be introduced. Firstly we will discuss the interpretation of nuclear diffuse scattering in binary systems where the two atomic species are perhaps not randomly mixed. Secondly, the technique of coherent-incoherent separation in nonmagnetic liquids and glasses will be considered.

\subsection{Nuclear Short-range order in binary systems}

Consider a randomly substituted binary alloy with a concentration $c$ of atoms of type $A$ having a scattering length of $b_{A}$, and a concentration $(1-c)$ of atoms of type B with a scattering length of $b_{B}$. From eq. 4.8 the incoherent scattering cross-section (excluding spin-incoherence) will be

$$
\begin{aligned}
\left(\frac{d \sigma}{d \Omega}\right)_{i n c} & =\overline{b^{2}}-(\bar{b})^{2} \\
& =c b_{A}^{2}+(1-c) b_{B}^{2}-\left(c b_{A}+(1-c) b_{B}\right)^{2} \\
& =c(1-c)\left(b_{A}-b_{B}\right)^{2}
\end{aligned}
$$

This is termed the Laue Monotonic Scattering cross-section of a binary atomic system, which will become large for a system with a large scattering length contrast $\left(b_{A}-b_{B}\right)$ (i.e. a large deviation of each of the individual scattering lengths from the mean).

Now, if the solution of $A$ and $B$ type atoms is non-random, then the eq. 4.2 will not hold, since

$$
\overline{b_{A} b_{B}} \neq \overline{b^{2}} \quad \forall \quad A=B
$$


unless the ensemble average is taken over a sufficiently long range. Let us restrict our attention to the average over the ensemble of individual near-neighbour shells of atoms, then we can write

$$
\begin{aligned}
\left(\overline{b_{A} b_{B}}\right)_{n} & =c b_{A}\left(p_{B}(n) b_{B}+\left[1-p_{B}(n)\right] b_{A}\right)+(1-c) b_{B}\left(p_{A}(n) b_{A}+\left[1-p_{A}(n)\right] b_{B}\right) \\
& =c b_{A}^{2}+(1-c) b_{B}^{2}-(1-c) p_{A}(n)\left(b_{A}-b_{B}\right)^{2}
\end{aligned}
$$

where $p_{B}(n)$ is the probability of finding a $B$ atom in the $n^{\text {th }}$ near-neighbour shell around an $A$ atom, and $p_{A}(n)$ is the probability of finding an $A$ atom in the $n^{t h}$ near-neighbour shell around a $B$ atom. The probablities are of course normalized such that $c p_{A}(n)=(1-c) p_{B}(n)$. The diffuse scattering in this case will be given by eq. 4.1 factorized into a sum over $n$ near-neighbour shells

$$
\begin{aligned}
\left(\frac{d \sigma}{d \Omega}\right)_{\text {diffuse }} & =\sum_{n=0}^{\infty}\left(\overline{b_{i} b_{j}}\right)_{n} Z_{n} \exp \left(i \vec{Q} \cdot \vec{R}_{n}\right)-(\bar{b})^{2} \\
& =c(1-c)\left(b_{A}-b_{B}\right)^{2} \sum_{n=0}^{\infty} Z_{n}\left(1-\frac{p_{A}(n)}{c}\right) \exp \left(i \vec{Q} \cdot \vec{R}_{n}\right)
\end{aligned}
$$

where $Z_{n}$ is the number of atoms in the $n^{\text {th }}$ near neighbour shell. This equation was derived separtely by Cowley [22] and Warren [23]. The term in brackets - which is the Fourier coefficient of the $n^{\text {th }}$ nearneighbour shell term in the Fourier sum, is termed the Warren-Cowley short-range order parameter

$$
\alpha_{n}=1-\frac{p_{A}(n)}{c}
$$

Warren-Cowley parameters have the following properties.

- For the zero ${ }^{\text {th }}$ near neighbour shell, the probability $p_{A}(0)=0$ and therefore, $\alpha(0)=1$

- For a random alloy, the probability $p_{A}$ (cf. of finding an $A$ atom around a $B$ atom) is simply equal to the concentration of $A$ atoms, $c$. Therefore, $\alpha(n>0)=0$, and eq. 6.5 reduces to the expression for the Laue monotonic scattering (eq. 6.1).

- The limiting values of the Warren-Cowley parameters are 1 ; representing a central $B$ atom entirely surrounded by like $B$ atoms and $(1-1 / c)$; for a central $B$ atom entirely surrounded by unlike $A$ atoms.

Eq. 6.5 can then be applied directly to the analysis of short-range order in single crystals. For the analysis of powders, we take the average over all directions of the position vector $\vec{R}_{n}$. This average is given by the integral over a spherical surface of radius $R_{n}$, i.e.,

$$
\begin{aligned}
\left\langle\exp \left(i \vec{Q} \cdot \vec{R}_{n}\right)\right\rangle & =\left\langle\exp \left(Q R_{n} \cos \phi\right)\right\rangle \\
& =\frac{1}{4 \pi R_{n}^{2}} \int_{0}^{2 \pi} \phi d \phi \int_{0}^{\pi} \exp \left(Q R_{n} \cos \theta\right) R_{n}^{2} \sin \theta d \theta \\
& =\frac{\sin Q R_{n}}{Q R_{n}}
\end{aligned}
$$

and the expression for the nuclear diffuse scattering becomes

$$
\left(\frac{d \sigma}{d \Omega}\right)_{\text {diffuse }}=c(1-c)\left(b_{A}-b_{B}\right)^{2}\left[1+\sum_{n=1}^{\infty} Z_{n} \alpha_{n} \frac{\sin Q R_{n}}{Q R_{n}}\right]
$$


Example 1: Nuclear short-range order in $\beta-M n A l$

The following example is taken from a recent study performed on D7, of a binary alloy, $\beta$-MnAl, which displays significant nuclear and magnetic short range order [24]. "xyz"- polarization analysis has been used to separate the nuclear, magnetic and spin-incoherent contributions to the total scattering, and the measured nuclear-only cross-sections are shown in Figure 3. The nuclear Bragg peaks have been removed from the data, leaving only the diffuse scattering contribution. Due to the fact that the $\beta$-Mn
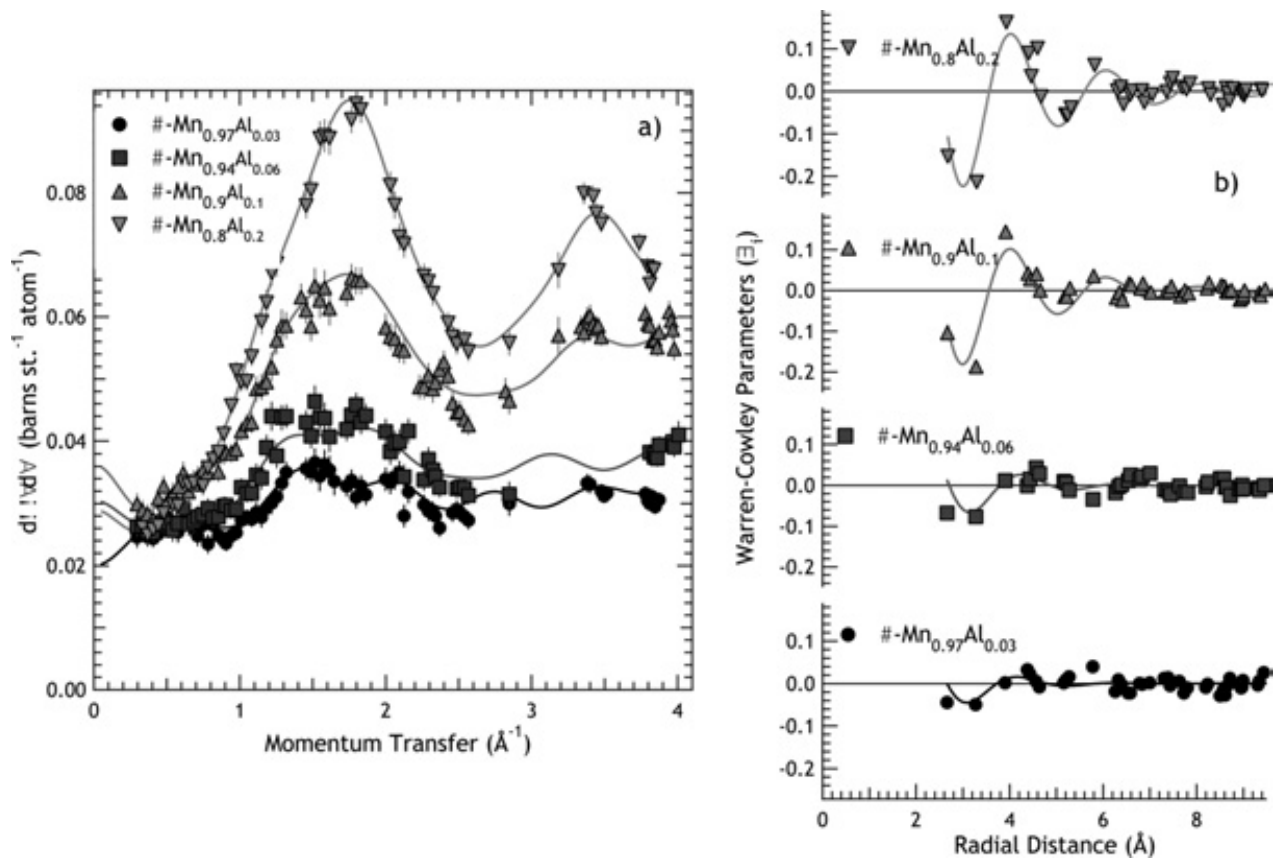

Figure 3. Nuclear substitutional short-range order in $\beta$-MnAl measured on D7. (a) The measured diffuse scattering cross-sections for the $\mathrm{Al}$ concentrations shown. The solid lines are RMC fits of eq. 6.8 to the data. (b) The calculated Warren-Cowley parameters for each Al concentration, deduced from the fits shown in (a) [24].

structure is non-centrosymmetric, there is a great number of near-neighbour shells at rather short distances from a central atom. In Figure 3(a) the solid lines are fits produced using a reverse monte carlo (RMC) procedure described in ref. [24], and in Figure 3(b) are the Warren-Cowley parameters (with respect to a central $\mathrm{Al}$ atom) deduced from these fits. We see that for all the $\mathrm{Al}$ concentrations shown, the first 2 near-neighbour parameters are negative, indicating that there is a less-than-average chance of finding an $\mathrm{Al}$ atom in these shells. The next few shells are positive, indicating a greater-than-average probability. Eventually, the oscillation around zero dies away, and the Warren-Cowley parameters converge to zero at large distances from the central atom - indicating random placement of $\mathrm{Al}$ atoms. The solid lines in Figure 3(b) are fits to a damped cosine function, $\cos (k r) \exp (-r / \xi)$, representing the socalled concentration-wave [25] of $\mathrm{Al}$ atoms in the lattice. The extent of the short-range substitutional order is given by the correlation length $\xi$, which is this case is found to increase steadily from around $1 \AA$ at low $\mathrm{Al}$ concentrations to around $2 \AA$ for large $\mathrm{Al}$ concentrations. 
Example 2: CuMn

CuMn has been studied for many years as an archetypal example of a canonical spin-glass system. Again, we see the presence of considerable diffuse scattering due to both substitutional short-range order of the $\mathrm{Mn}$ atoms on the $\mathrm{Cu}$ lattice, and short-range magnetic correlations. In this particular case both the magnetic and nuclear diffuse scattering is seen to be peaked around the $\left(10 \frac{1}{2}\right)$ lattice positions - so in this case, polarization analysis is essential to separate the nuclear and magnetic contributions. The nuclear diffuse scattering from a single crystal of $\mathrm{Cu}-25 \mathrm{at} \% \mathrm{Mn}$ is shown in Figure 4. The nuclear

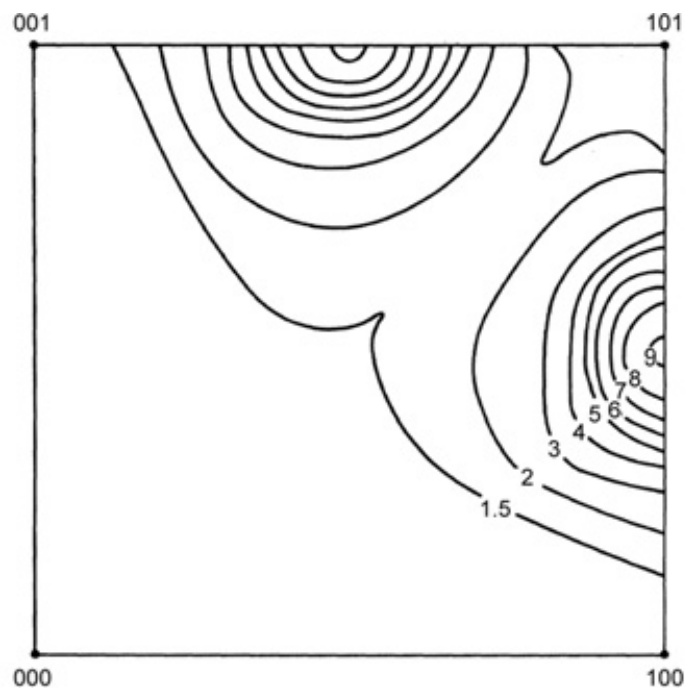

Figure 4. The nuclear scattering from the [h 0 l] of $\mathrm{Cu}-25$ at $\% \mathrm{Mn}$ taken from ref [26]. Diffuse intensity is heaped up around the $\left(\begin{array}{lll}1 & 0 & \frac{1}{2}\end{array}\right)$ and $\left(\frac{1}{2} 01\right)$ positions.

diffuse scattering in this case (and in the previous case of $\beta$-MnAl) is strong, since the scattering length contrast $b_{C u}-b_{M n}$ is large (N.B., Mn has a negative scattering length) Fits of this data to Eq. 6.5 showed an oscillatory behaviour of the Warren-Cowley parameters, indicating that the Mn atoms displayed a strong tendency to anticluster; i.e. they tend to position themselves wide apart in the $\mathrm{Cu}$ lattice.

\subsection{Coherent/Incoherent Separation in non-magnetic systems}

Measurement of the static structure factors of polymer liquids and glasses can often be complicated by the presence of large amounts of hydrogen. The spin-incoherent scattering cross-section of hydrogen is huge : 80.3 barns per hydrogen atom compared with a coherent scattering cross-section of only 1.65 barns per hydrogen atom. In polymer systems then, the spin-incoherent scattering cross-section dominates the neutron diffraction pattern, and extraction of the coherent static structure factor is difficult ; requiring precise knowledge of the abundance of hydrogen in the sample so that the (almost) flat spin-incoherent scattering level may be subtracted from the data. In practice this is very difficult to accomplish. Another approach would be to deuterate the sample (the spin-incoherent scattering crosssection of deuterium is only 2.05 barns per deuterium atom) but this normally alters the static structure factor of the polymer as well as the dynamical properties. Polarized neutrons therefore provide the most reliable way of extracting the coherent static structure factor in polymer systems. 
In addition, if the abundance of hydrogen in the sample is well known, then the flat spin-incoherent scattering can be used to normalize the scattering spectra, so that they may be put on an absolute scale (barns st ${ }^{-1}$ atom $^{-1}$ ) - a procedure which normally requires the measurement of a standard vanadium sample. In this way systematic errors (due to sample size and shape effects) are much reduced. This procedure is known as inter-normalization. It should be emphasized that inter-normalization also corrects the data for inelastic scattering at high $\mathrm{Q}$, since the spin-incoherent scattering provides a direct measurement of the Debye-Waller factor. For a review of polarized neutron scattering studies of polymers, see the article by Tölle [27].

Example 1: Partial structure factors of polyisoprene

In this example, polarized neutrons were used to extract the partial structure factors in polyisoprene which consists of a methyl group on a polymer chain [28]. Four samples were studied ; fully protonated, fully deuterated, methyl group deuterated and main-chain deuterated, in order to extract the partial structure factors. The inter-normalized neutron diffraction patterns are shown in Figure [5]. The level of
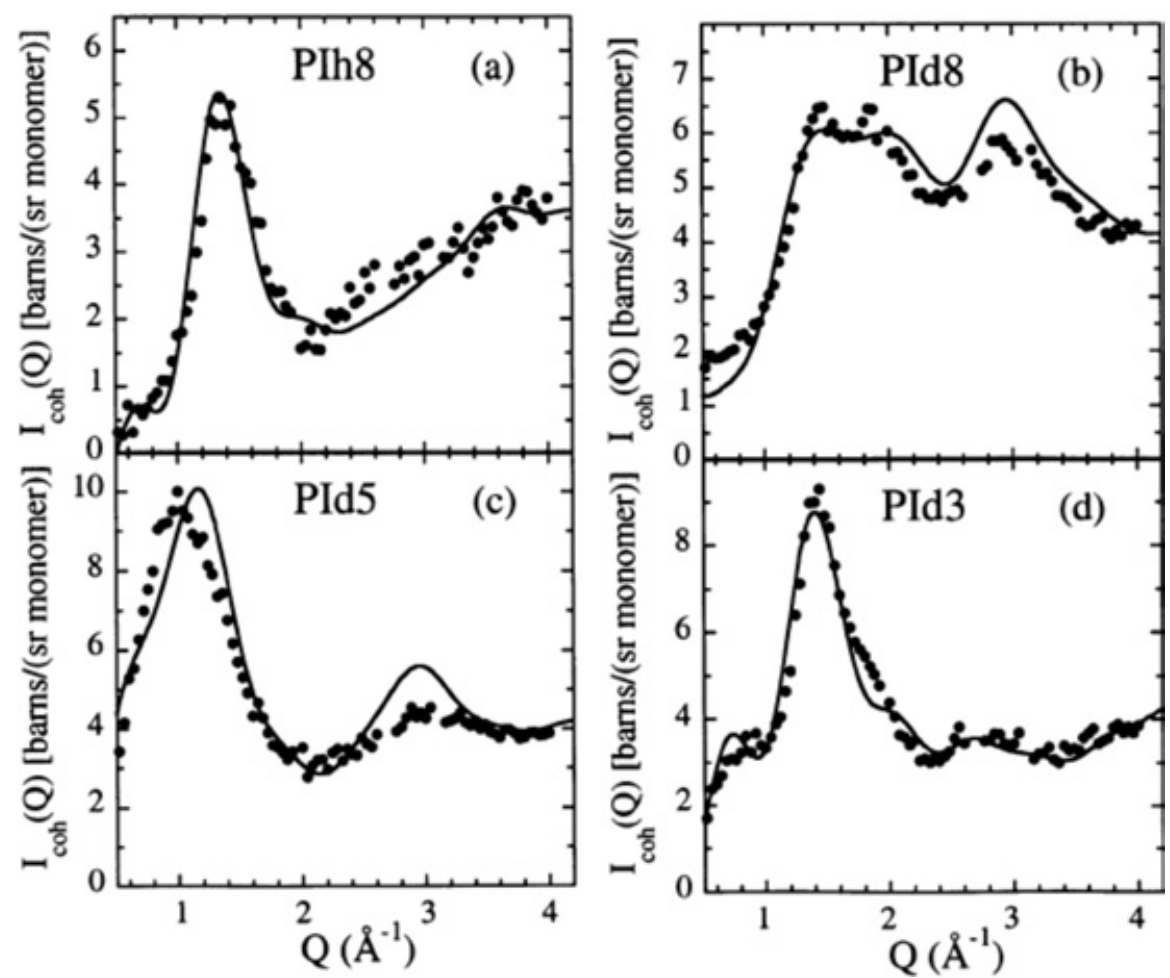

Figure 5. Differential coherent scattering cross-sections of the four deuterations of polyisprene (PIh8 - fully protonated, PId8 - fully deuterated, PId5 - main-chain deuterated, and PId3, methyl group deuterated). Solid lines correspond to molecular dynamics simulations [28].

agreement between the fully corrected and normalized experimental data and the molecular dynamics simulations is remarkable - resulting in a accurate physical picture of the intra- and inter-chain correlations in the polymer. 
Example 2: Inelastic scattering in para- $\mathrm{H}_{2}$

Inelastic polarization analysis measurements of fluctuations in disordered molecular systems or quantum fluids such as ${ }^{3} \mathrm{He}$ or $\mathrm{H}_{2}$ can give essential information on the origin (collective or single particle) of the dynamics. The spin-incoherent inelastic scattering will be entirely due to single particle effects, while the coherent scattering is due solely to cooperative excitations. To date, the only instrument, worldwide, which can perform inelastic time-of-flight measurements of these excitations is D7. Figure 6 shows the
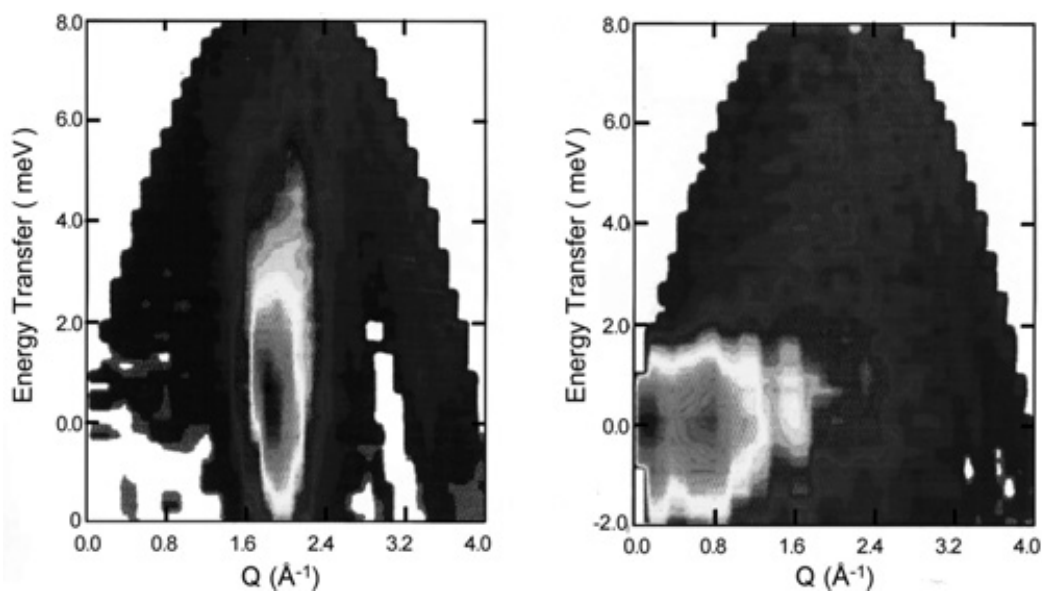

Figure 6. The coherent (left) and spin-incoherent (right) $S(Q, \omega)$ of liquid para-hydrogen at $16 \mathrm{~K}$ measured simultaneously using time-of-flight polarization analysis on D7, with an incident energy of 9 meV [29].

coherent and spin-incoherent $S(Q, \omega)$ of liquid para-hydrogen at $16 \mathrm{~K}$ measured simultaneously using time-of-flight polarization analysis on D7 [29]. These spectra have been analysed in terms of self and distinct correlations in the molecular liquid.

\section{MAGNETIC DIFFUSE SCATTERING}

In this section we look at the diffuse scattering from magnetic systems. These include long-range ordered (ferromagnetic or antiferromagnetic) materials with defects (overlapping or otherwise), as well as disordered magnets such as paramagnets and spin-liquids and glasses.

\subsection{Defect scattering in ferromagnets}

Consider a fully ordered ferromagnetic lattice of $A$ atoms, and introduce a localised magnetic defect atom $B$. The magnetic scattering lengths of the $A$ and $B$ atoms are given by

$$
p_{A, B}=\left(\frac{\gamma_{n} r_{0}^{2}}{2}\right)\left\langle\mu_{A, B}\right\rangle f_{A, B}(Q)[\vec{P} \cdot \vec{q}]
$$

where $\vec{q}$ is the so-called magnetic interaction vector $\vec{q}=\widehat{Q}(\widehat{Q} \cdot \widehat{\eta})-\widehat{\eta}$ where $\eta$ is the magnetization direction of the ferromagnet. This is related to the transverse magnetization operator by, $\vec{M}_{\perp}=2 \mu_{B} \vec{q}$. In direct analogy to the expression for the Laue monotonic scattering, eq. 6.1, we can write an expression for the magnetic Laue monotonic scattering caused by the introduction of the magnetic defect. In the case of unpolarized neutrons we have for the magnetic scattering cross-section 


$$
\left(\frac{d \sigma}{d \Omega}\right)_{M a g}=c(1-c) q^{2}\left(\frac{\gamma_{n} r_{0}^{2}}{2}\right)^{2}\left[\mu_{A} f_{A}(Q)-\mu_{B} f_{B}(Q)\right]^{2}
$$

Note that in the case of a randomly oriented multi-domain ferromagnet (or, indeed, a paramagnet) we would have for the magnetic interaction vector

$$
q^{2}=1-(\widehat{Q} \cdot \widehat{\eta})^{2}=\frac{2}{3}
$$

In the case of an extended defect, i.e. one which alters the magnetic moment on atoms in its vicinity, the expression for the magnetic diffuse scattering becomes

$$
\left(\frac{d \sigma}{d \Omega}\right)_{M a g}=c(1-c) q^{2}\left(\frac{\gamma_{n} r_{0}^{2}}{2}\right)^{2}\left[\mu_{A} f_{A}(Q)-\mu_{B} f_{B}(Q)+\Phi(Q)\right]^{2}
$$

where $\Phi(Q)$ is the Fourier transform of the defect magnetisation density $M(r)$

$$
\Phi(\vec{Q})=\sum_{r} \exp (i \vec{Q} \cdot \vec{r}) M(r)
$$

In the case of a polarized incident beam, there is an additional polarization dependent cross term [30] which appears,

$$
\left(\frac{d \sigma}{d \Omega}\right)_{p o l}=\left(\frac{\gamma_{n} r_{0}^{2}}{2}\right)(\vec{P} \cdot \vec{q}) c(1-c)\left(b_{A}-b_{B}\right)\left[\mu_{A} f_{A}(Q)-\mu_{B} f_{B}(Q)+\Phi(Q)\right]
$$

This additional term may be separated from the pure magnetic and pure nuclear scattering simply by taking the difference between the cross-sections measured with a spin-up $(\vec{P}=1)$ and spin-down $(\vec{P}=-1)$ incident polarization direction. The main advantage over the unpolarized beam method is therefore that the sign of the the moment disturbance $\left(\mu_{A}-\mu_{B}\right)$, is determined. However, we also note that in order to obtain a good difference signal, we need good magnetic and nuclear contrast. I.e. the difference $\left(b_{A}-b_{B}\right)$ should be large. Another great advantage of the polarized neutron method is that it is straightforward to place the difference scattering on an absolute scale, which therefore becomes comparable with bulk magnetization measurements. The bulk magnetization per atom is

$$
\bar{\mu}=c \mu_{A}+(1-c) \mu_{B}
$$

Therefore, the defect concentration dependence of the bulk magnetization is given by

$$
\frac{d \bar{\mu}}{d c}=\left(\mu_{A}-\mu_{B}\right)
$$

and in the limit of $Q \rightarrow 0$ we can therefore write

$$
2\left(\frac{d \sigma}{d \Omega}\right)_{p o l}=\left(\frac{d \sigma}{d \Omega}\right)_{+}-\left(\frac{d \sigma}{d \Omega}\right)_{-}=2\left(\frac{\gamma_{n} r_{0}^{2}}{2}\right) c(1-c)\left(b_{A}-b_{B}\right)\left(\frac{d \bar{\mu}}{d c}\right)
$$

The difference scattering extrapolated to $Q=0$ may therefore be directly compared to bulk magnetization measurements, and hence the separate magnetic moments $\mu_{A}$ and $\mu_{B}$ can be readily determined.

The above treatment is valid for systems in the dilute defect limit. For more concentrated alloys the treatment is more complicated since defects can overlap. In addition, moment perturbations can be found on both atomic species. This case was described by Marshall [31], using a model assuming linear superposition of defects, and examples are given in the review of Rainford [30]. 
Example: Magnetic defects in ferromagnetic Fe

Mezei and co-workers used polarized neutrons on D7 to investigate the extended magnetic defects introduced into ferromagnetic iron on substitution of dilute quantities of magnanese. Figure 7 shows the
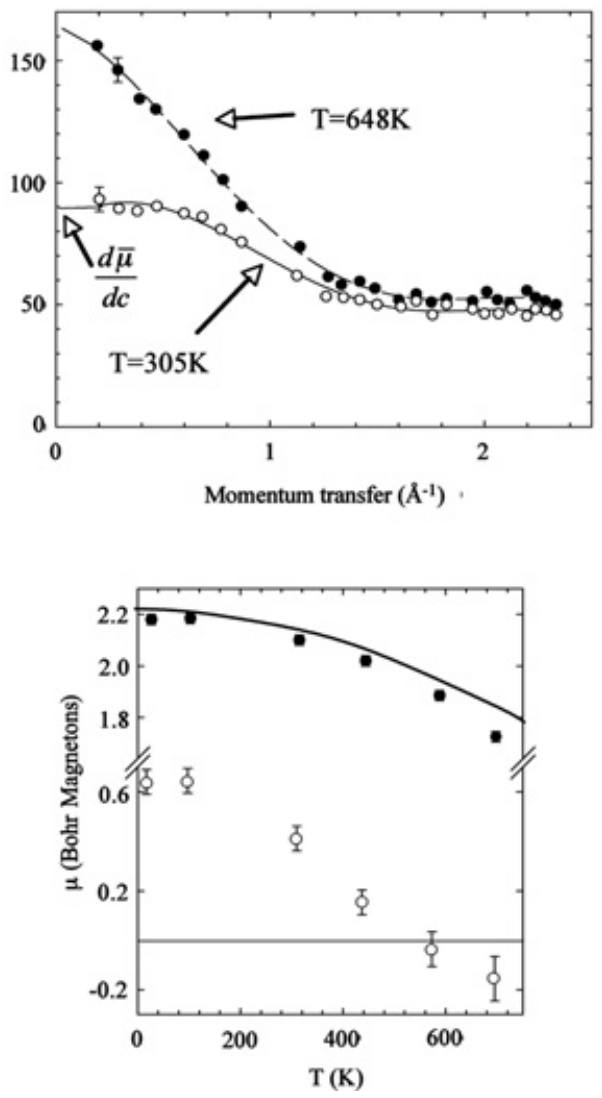

Figure 7. Top : polarized difference cross-section of $\mathrm{Fe}-2.8 \mathrm{at} \% \mathrm{Mn}$ at two temperatures. Bottom : the temperature dependence of the magnetic moments on the Mn (open symbols) and surrounding iron atoms (solid symbols), compared to the pure iron moment (solid line).

polarized difference cross-section of $\mathrm{Fe}_{0.972} \mathrm{Mn}_{0.028}$ measured at two temperatures. These were analysed using the powder averaged from of eq. 7.6 and with the approximation that $f_{F e} \approx f_{M n}$, i.e.

$$
\Delta\left(\frac{d \sigma}{d \Omega}\right)=2\left(\frac{\gamma_{n} r_{0}^{2}}{2}\right) c(1-c)\left(b_{A}-b_{B}\right) f_{F e}(Q)\left[\mu_{A}-\mu_{B}+\sum_{n} z_{n} \delta \mu_{n} \frac{\sin Q r_{n}}{Q r_{n}}\right]
$$

where the last term in the square brackets is the powder average of the extended defect (eq. 7.5), which extends over $n$ near-neighbour shells. In this case, the data were fitted assuming a defect extending over the first two near-neighbour shells only. The lower plot in Figure 7 shows the inferred Mn moment and the 1st near-neighbour shell iron moment $=\mu_{P u r e F e}-\delta \mu$, as well as the pure iron moment. 


\subsection{Defect scattering in Antiferromagnets}

Unlike the case of ferromagnetic binary systems, it is not generally possible to affect the orientation of the magnetic moments by the application of a magnetic field, and therefore the polarization switching technique cannot be applied. However, as long as there are no chiral components to the magnetic structure, "xyz"- polarization analysis may be used to extract the pure magnetic cross-section (see section 5.2). If we assume that we have a single domain antiferromagnet with a known magnetization direction, $\widehat{\eta}$, then the magnetic diffuse cross-section is given by

$$
\left(\frac{d \sigma}{d \Omega}\right)_{M a g}=\left(\frac{\gamma_{n} r_{0}^{2}}{2}\right)^{2} q^{2} c(1-c) S(\vec{Q}-\vec{\tau})\left[\nu_{A} f_{A}(Q)-\nu_{B} f_{B}(Q)+\Phi(\vec{Q}-\vec{\tau})\right]^{2}
$$

where $\nu_{A}$ and $\nu_{B}$ are the sublattice moments of each species and $\vec{\tau}$ is the antiferromagnetic propagation vector such that $\exp \left(i \vec{\tau} \cdot \vec{R}_{i}\right)=1$ for $\vec{R}_{i}$ on the up sublattice and -1 for the down sublattice. In the case where we have a multi-domain antiferromagnet (such as an antiferromagnetic powder), then the factor $q^{2}=\frac{2}{3}$, from eq. 7.3. In general, analysis of spherically averaged data can be complicated, since there will be contributions to the scattering from more than one Brillouin zone (see for example, [30]).

\section{Example: Fe defects in chromium}

Pure chromium is an incommensurate spin-density-wave (SDW) antiferromagnet at room temperature. The substitution of dilute $\mathrm{Fe}$ impurities results in a rapid decrease of the magnetic transition temperature and the mean magnetic moment [32]. Figure 8 shows the magnetic and nuclear diffuse scattering of Cr-6.5at.\% Fe, modelled according to eqs. 7.11 and 6.8 respectively [33]. The form of $S(\vec{Q}-\vec{\tau})$ found from the nuclear diffuse scattering (which indicates a tendency towards clustering of the Fe atoms) was substituted into eq. 7.11 which was then, in turn, used to model the magnetic data. The moment

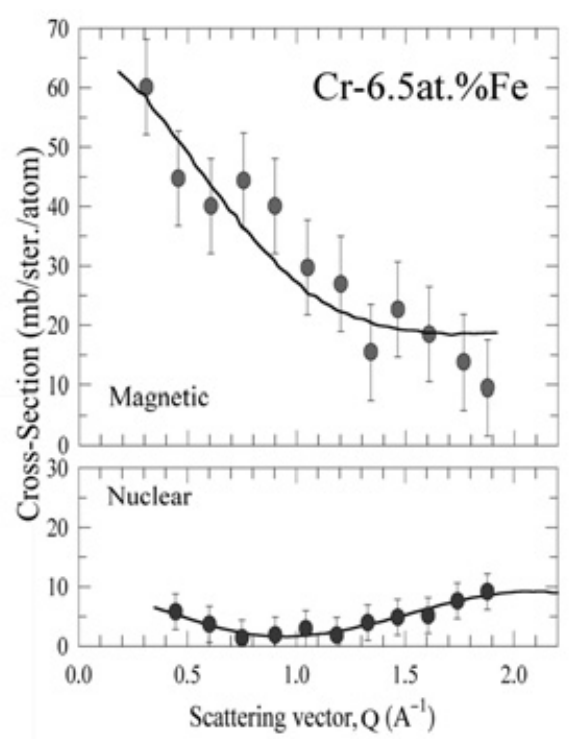

Figure 8. The magnetic (top) and nuclear (bottom) diffuse scattering in Cr-6.5at.\% Fe measured on the LONGPOL diffuse scattering spectrometer at ANSTO [2]. The magnetic data are modelled according to eq. 7.11 with the form of $S(\vec{Q}-\vec{\tau})$ found by fitting the nuclear data as shown. 
contrast, $\nu_{F e}-\nu_{C r}=-0.6 \pm 0.08$ and since magnetisation indicates $\nu_{C r} \sim 0.68$, the Fe moment is reduced almost to zero. In addition, the moment disturbances on the first two near neighbours are found to be $\Delta \nu\left(r_{1}\right) \sim 0.58$ and $\Delta \nu\left(r_{2}\right) \sim-0.55$. Therefore, the amplitude of the $\mathrm{Cr} \mathrm{SDW}$ is reduced almost to zero up to two near-neighbours away from the Fe defect atoms.

\subsection{Paramagnetic and disordered spin systems}

It is in the study of disordered systems such as spin glasses and paramagnets that neutron polarization analysis comes into its own. The magnetic cross section from such systems is always diffuse and often weak, so that a proper separation of the magnetic and nuclear cross-section can only be achieved with neutron polarization analysis. In a paramagnetic system, or a static disordered magnet (spin-glass) with one magnetic species, we can write the diffuse magnetic cross-section as

$$
\left(\frac{d \sigma}{d \Omega}\right)_{M a g}=q^{2}\left(\frac{\gamma_{n} r_{0}^{2}}{2}\right)^{2} f^{2}(\vec{Q}) S(\vec{Q}) M^{2}(\vec{Q})
$$

where $M(\vec{Q})$ is the Fourier transform of the spatially varying magnetization density $M(\vec{r}) \cdot M^{2}(\vec{Q})$ can be written as

$$
M^{2}(\vec{Q})=g_{S}^{2} S(S+1) \sum_{n} \frac{\left\langle\vec{S}_{0} \cdot \vec{S}_{n}\right\rangle}{S(S+1)} \exp \left(i \vec{Q} \cdot \vec{r}_{n}\right)
$$

where $\vec{S}_{0}$ and $\vec{S}_{n}$ are the atomic spin vectors at the origin and the $\mathrm{n}^{\text {th }}$ nuclear lattice position. Factorizing eq. 7.13 into near neighbour shells, and taking the powder average, we arrive at the expression

$$
\left(\frac{d \sigma}{d \Omega}\right)_{M a g}=\frac{2}{3}^{2} f^{2}(\vec{Q}) g_{S}^{2} S(S+1)\left[1+\sum_{N} \frac{\left\langle\vec{S}_{0} \cdot \vec{S}_{N}\right\rangle}{S(S+1)} Z_{N} \frac{\sin Q r_{N}}{Q r_{N}}\right]
$$

where $r_{N}$ and $Z_{N}$ are the radius and coordination number of the $\mathrm{N}^{t h}$ near-neighbour shell, and where we have implicitly assumed that there are no concentration driven fluctuations in the magnetic moment distribution arising from nuclear short-range order, so that $S(\vec{Q})=1$ in eq. 7.12. In cases where this assumption cannot be made, the calculation of the spherical average of $f^{2}(\vec{Q}) S(\vec{Q}) M^{2}(\vec{Q})$ must be made explicitly. In all but the simplest structures, (see for example [33]) this calculation is very difficult, and numerical modeling methods must be used to take full account of the nuclear and magnetic contributions to the magnetic diffuse cross-section.

In order to properly characterize the spin-correlations in an intrinsically dynamic system such as a spin glass or paramagnet, it is essential that the experiment be performed within the so-called quasi-static approximation, that is, the inelasticity of the scattering due to spin fluctuations must be small compared to the incident energy of the neutrons and the thermal energy $k_{B} T$ must be greater than the spectral width of the spin fluctuations. This condition is not always satisfied in diffuse scattering investigations since one of the general requirements of a diffuse scattering instrument is long wavelength (cold) neutrons, so that diffuse intensity from multiple Bragg scattering is minimized. Furthermore, the supermirror bender neutron polarization analysers used on D7, DNS and LONGPOL have a limited energy range since their transmission drops sharply as the neutron energy increases. For spin glass alloys, the quasi-static approximation is usually well satisfied [34] since at low temperatures the spins are in a quasistatic ground state, and the response is therefore essentially elastic. However, studies of itinerant topologically frustrated magnets have shown, that there may be a substantial spin fluctuational spectral width well below the apparent spin glass freezing temperature. For example, measurements by Cywinski and co-workers 
on the cubic Laves phase compound $\mathrm{Y}\left(\mathrm{Mn}_{0.95} \mathrm{Fe}_{0.05}\right)_{2}$ using polarization analysis revealed strong antiferromagnetically correlated spin fluctuations with a mean moment of $0.2 \mu_{B}$ per $\mathrm{Mn} / \mathrm{Fe}$ atom [35]. This compares with a value of $2 \mu_{B}$ per $\mathrm{Mn} / \mathrm{Fe}$ atom measured using high-energy neutron inelastic scattering [36]. It is essential therefore, that the spectral width of the spin fluctuations in itinerant systems is well characterized so that it is clear that the measurements are being carried out within the quasistatic approximation.

\section{Example 1: Paramagnetic correlations in $\mathrm{MnO}$}

$\mathrm{MnO}$ is well known to be a model Heisenberg antiferromagnet with a transition temperature of $T_{N}=$ $120 \mathrm{~K}$. Studies of $\mathrm{MnO}$ magnetic neutron diffraction, were amongst the first ever performed [37,38]. More recent studies of $\mathrm{MnO}$ single crystals have shown that short-range order of the $\mathrm{Mn}^{2+}$ ions persists to high temperatures (around $6 \times T_{N}$ ). This short-range order has been studied by Hohlwein and co-workers [39], and analysed in terms of a mean-field model of the staggered susceptibility. However, since Hohlwein used unpolarized neutrons, his signal was contaminated with nuclear an incoherent scattering. Figure 9 shows the pure magnetic diffuse scattering of $\mathrm{MnO}$ in the (h $\mathrm{h} \mathrm{l}$ ) crystal plane measured

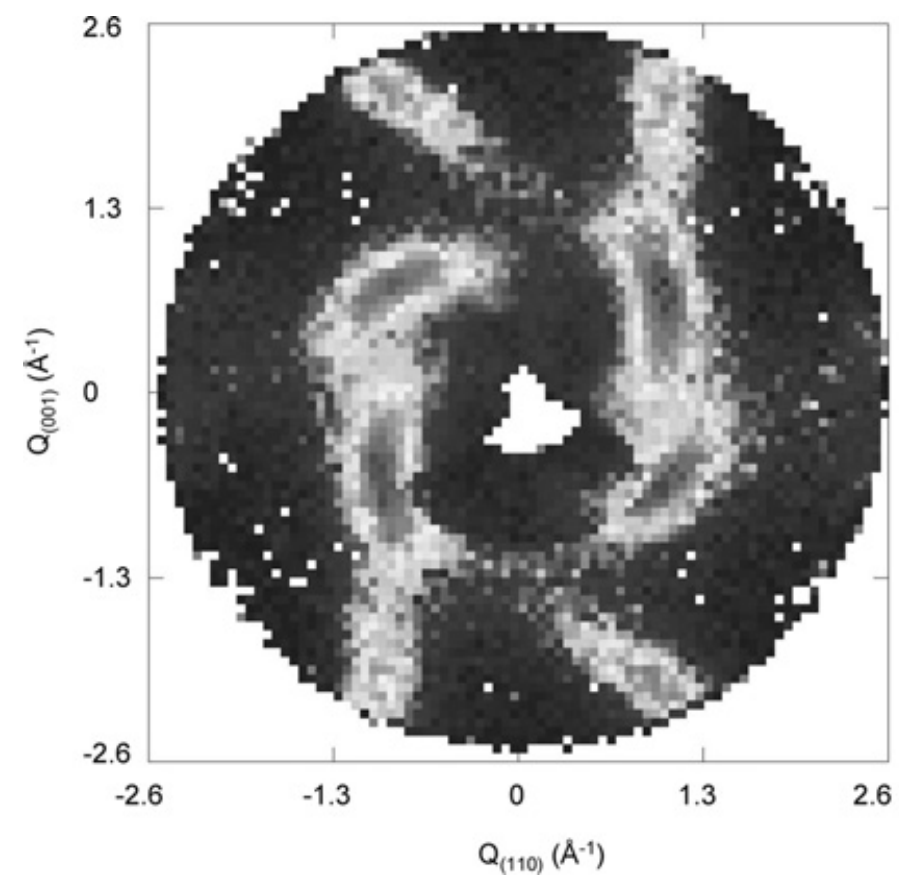

Figure 9. The magnetic diffuse scattering cross-section of $\mathrm{MnO}$ in the (h h l) plane at $150 \mathrm{~K}$, measured on D7 using polarization analysis. The data show diffuse peaks around the eventual AF Bragg peak positions, but the 4fold cubic symmetry has been lowered to 2-fold symmetry.

using polarized neutrons on D7. This data is in the process of been analysed, but preliminary results seem to indicate a possible imbalance of antiferromagnetic short-range domains. This is indicated by the fact that the 4-fold cubic symmetry present in the ordered phase, has been lowered to 2-fold symmetry of the diffuse magnetic peaks. Studies of this sort, on small single crystals with full crystal plane mapping, have only recently become possible due to major improvements in the D7 spectrometer detector bank [40]. 
Example 2: Magnetic structure of $\mathrm{Gd}_{2} \mathrm{Ti}_{2} \mathrm{O}_{7}$ deduced from diffuse scattering

Occasionally, the analysis of the diffuse scattering of a partially disordered magnet can lead to the elucidation of the magnetically ordered component. A good example of this is $\mathrm{Gd}_{2} \mathrm{Ti}_{2} \mathrm{O}_{7}$. This is a geometrically fully frustrated magnet, due to dominant antiferromagnetic correlations on a tetrahedrally coordinated pyrochlore lattice (for a review of fully frustrated magnets and $\mathrm{Gd}_{2} \mathrm{Ti}_{2} \mathrm{O}_{7}$ in particular, see [42]). Two low temperature magnetic transitions (at $1 \mathrm{~K}$ and $0.7 \mathrm{~K}$ ) have been identified using conventional neutron diffraction and specific heat studies. However, due to the difficulty of differentiating between single-k and multi-k magnetic structures (see [41]), it was impossible to decide between two different magnetic ground states from the analysis of the conventional powder neutron diffraction studies. The two alternative structures proposed in [43] are depicted in Figure 10. In the 1k structure (left)
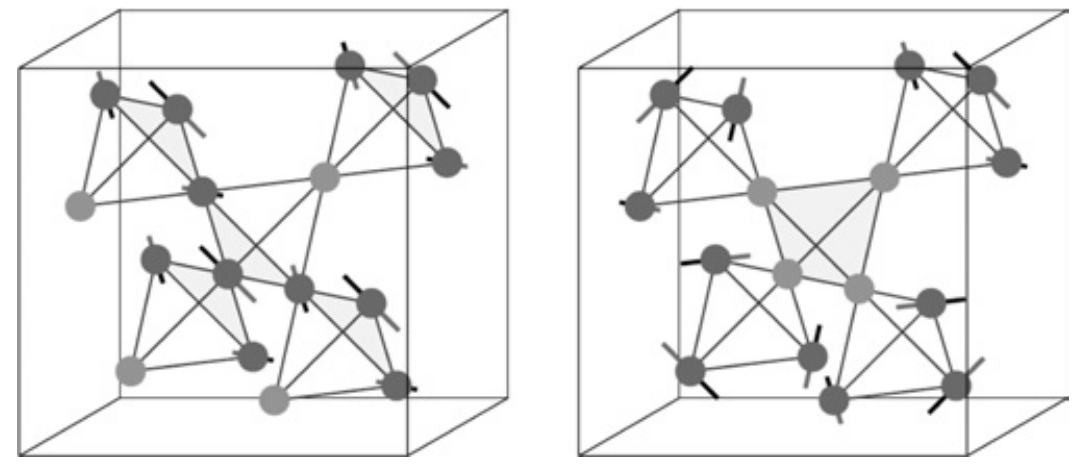

Figure 10. The two possible low temperature magnetic structures of $\mathrm{Gd}_{2} \mathrm{Ti}_{2} \mathrm{O}_{7}$. The so-called " $1 \mathrm{k}$ " structure on the left, and the " $4 \mathrm{k}$ " structure on the right. The Gd atoms coloured orange do not participate in the ordered structure, and hence would be expected to produce diffuse scattering [44].

the ordered Gd spins are all perpendicular to a global [ $\left[\begin{array}{lll}1 & 1 & 1\end{array}\right]$ axis, in a quasi-2d kagomé type plane. In the $4 \mathrm{k}$ structure (right) the Gd ordered spins are perpendicular to local trigonal $<111>$ axes connecting the vertices of each tetrahedron to its centre. It is worth noting that the disordered Gd spins (coloured orange in Figure 10) differ in respect of their local coordinations in the two different structures, with the near-neighbour Gd-Gd distance in the $1 \mathrm{k}$ disordered lattice $(7.4 \AA)$ being much larger than that of the $4 \mathrm{k}$ disordered lattice (3.6 $\AA$ ), in which the whole central tetrahedron is disordered. The diffuse magnetic scattering due to the disordered Gd atoms has been measured on D7 [44] and is shown in Figure 11.

Using a argument based on "naive inversion", differentiation of eq. 7.14 gives and estimate of the position of the first structure factor peak at $Q_{\text {peak }}=4.5 / R_{1}$. Using this argument, we would expect to find the first structure factor peak at $Q_{\text {peak }} \sim 0.61 \AA^{-1}$ for the $1 \mathrm{k}$ model and at $Q_{\text {peak }} \sim 1.2 \AA^{-1}$ for the $4 \mathrm{k}$ model. The data seem to support the $4 \mathrm{k}$ model with the first structure factor peak coming at $Q \approx 1.1 \AA^{-1}$. To be sure of the model, the data have been fitted as shown in figure 11 to eq. 7.14 using the first two near neighbour shells. It is clear that the $4 \mathrm{k}$ disordered structural model is much closer to the data than the $1 \mathrm{k}$ model. In addition, the absolute normalization of the data made possible with polarized neutrons has confirmed the proportion of ordered to disordered $\mathrm{Gd}$ atoms in the magnetic ground state [44]. This is one of the rare examples of the elucidation of the magnetic disordered structure providing valuable evidence as to the nature of the ordered magnetic ground state.

\section{Example 3: Nuclear and magnetic short-range order in $\beta$-Mn alloys}

In section 6.1.1 we saw that the introduction of dilute amounts of $\mathrm{Al}$ into the simple cubic $\beta$-Mn allotrope, results in a non-random anticlustering arrangement of the $\mathrm{Al}$ atoms on the Mn lattice. We now 


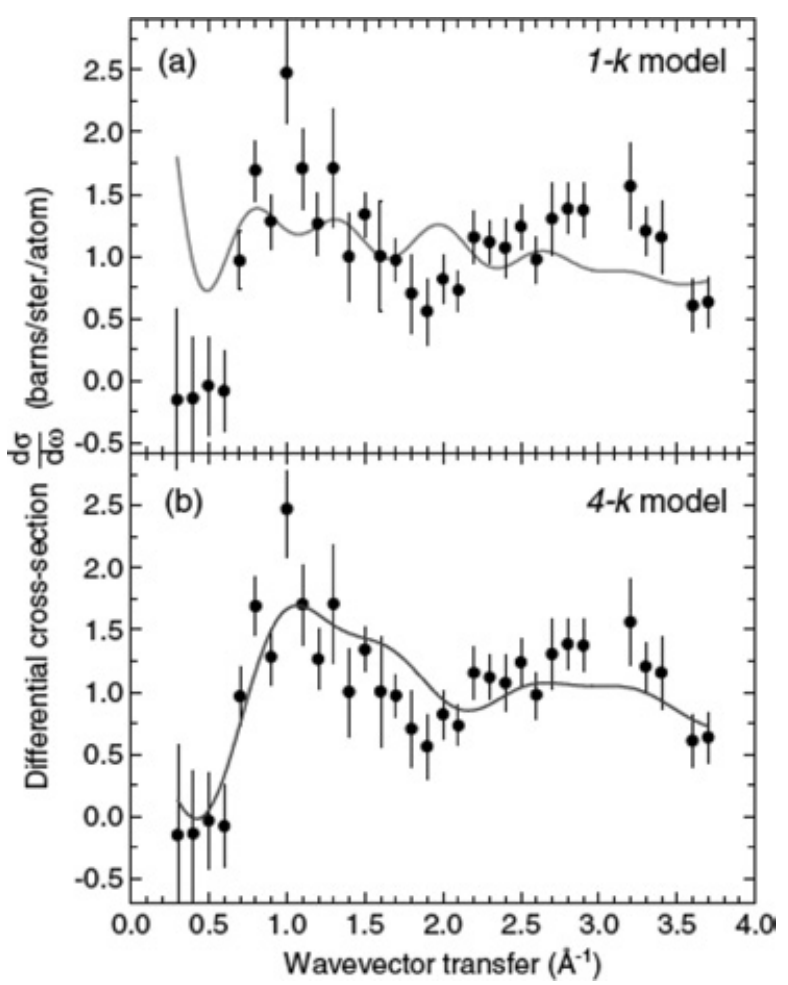

Figure 11. Magnetic diffuse scattering of $\mathrm{Gd}_{2} \mathrm{Ti}_{2} \mathrm{O}_{7}$ measured on $\mathrm{D} 7$ at $46 \mathrm{mK}$. Solid lines show fits of the data to the disordered component of (a) the 1-k and (b) the 4-k structures (see Figure 10) using equation 7.14, using two near neighbour shells $(\mathrm{N}=1,2)$ and three adjustable parameters: $\left\langle\vec{S}_{0} \cdot \vec{S}_{1}\right\rangle,\left\langle\vec{S}_{0} \cdot \vec{S}_{2}\right\rangle$ and $S(S+1)$ [44].

turn our attention to the magnetic structure of $\beta$-MnAl. Pure $\beta$-Mn is a frustrated spin-liquid, showing no magnetic order down to low temperatures, but displaying a range of interesting properties, including a large electronic component of the specific heat $\left(\gamma=80 \mathrm{~mJ} \mathrm{~mol}^{-1} \mathrm{~K}^{-2}\right)$ and non-Fermi-liquid scaling of paramagnetic spin fluctuations [45,46]. The addition of $\mathrm{Al}$ impurities (as well as $\mathrm{Co}, \mathrm{Fe}$, In, etc) has been shown to result in a so-called spin-liquid to spin-glass transition $[45,47]$. The lack of long-range magnetic order in $\beta$-Mn is thought to be due to the presence of geometrical frustration in the $\beta$-Mn lattice (although the $\beta-\mathrm{MnRu}$ alloy with $\mathrm{Ru}$ concentrations of $>12$ at. \% has been recently reported to possess a long-range ordered magnetic ground state [48]). The magnetic diffuse scattering of several $\beta-\mathrm{MnAl}$ alloys, measured simultaneously with the nuclear diffuse scattering presented in Figure 3 , is shown in Figure 12 [24].

The data have been modelled according to eq. 7.14 using the same RMC procedure that was adopted for the analysis of the nuclear structure. The usefulness of the RMC approach should be stressed here. Using a physical model of the Al-substituted Mn lattice extracted from the nuclear data, any concentration driven effects that may feed through into the magnetic diffuse scattering are automatically taken into account. Furthermore, the near neighbour correlation parameters, $\left\langle\vec{S}_{0} \cdot \vec{S}_{N}\right\rangle$ are not freely adjustable independent parameters. They are, in fact, highly correlated. This is easily seen from the observation that a strong antiferromagnetic correlation in the first near-neighbour shell normally leads to a strong ferromagnetic correlation at twice that distance. The oscillatory behaviour of the magnetic correlations 

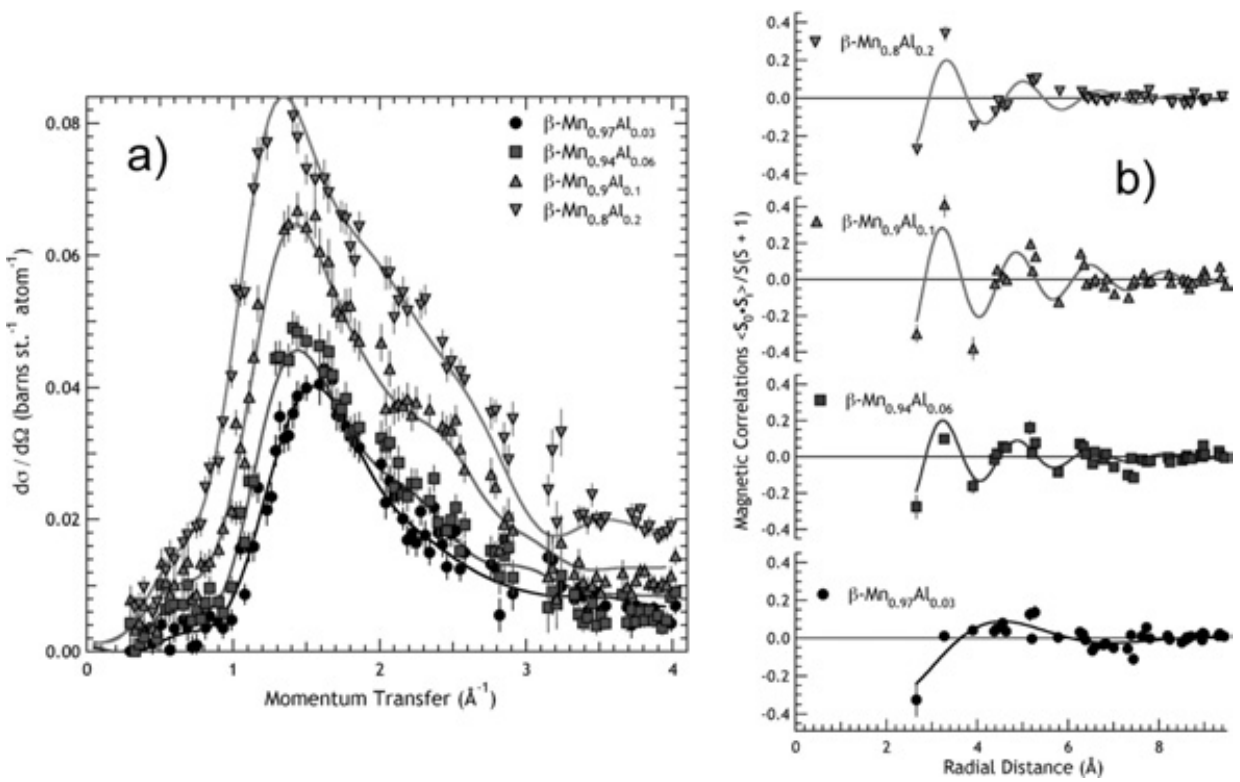

Figure 12. Magnetic short-range order in $\beta$-MnAl measured on D7. (a) The measured diffuse scattering crosssections for the $\mathrm{Al}$ concentrations shown. The solid lines are RMC fits of eq. 7.14 to the data. (b) The calculated magnetic correlation parameters for each Al concentration, deduced from the fits shown in (a)[44].

is qualitatively similar in all four $\beta$-MnAl compositions studied, with the $1^{\text {st }}$ and $3^{\text {rd }}$ near neighbour shells antiferromagnetically correlated, and the $2^{\text {nd }}$ near neighbour shell ferromagnetically correlated. The radius of the second shell of $3.27 \AA$ corresponds to a momentum transfer of $Q_{\text {peak }} \sim 1.4 \AA^{-1}$, where we see a large peak in the experimental cross-section. A broad region of ferromagnetic correlations is observed centred at around $R=5.2 \AA$ corresponding to $Q_{\text {peak }} \sim 1.2 \AA^{-1}$. This peak in the magnetic correlation parameters falls steadily with increasing $\mathrm{Al}$ concentration indicating that the correlations are of longer range in the more dilute $\beta$-MnAl alloys. This is borne out by the fact that the magnetic diffuse scattering becomes increasingly broad as the Al concentration increases. In addition, since the first three near neighbour correlations increase in magnitude on increasing Al concentration it is likely that the frustration inherent in the $\beta$-Mn lattice is being disrupted by the introduction of $\mathrm{Al}$ atoms, therefore facilitating the alignment of the neighbouring Mn spins.

\section{SUMMARY}

This article has presented some applications of neutron polarization analysis in the field of disorder scattering in crystalline (and polymer glass/liquid) materials. The analysis of diffuse scattering is notoriously difficult, due to the number of different possible reasons that exist which could possibly cause it. The use of polarized neutrons is therefore an essential prerequisite so that the contributions to the diffuse scattering may be separated out as far as is possible, and then analysed separately. Since diffuse scattering is always weak, and polarized neutron instruments are always lower in flux than their unpolarized counterparts, the measurement of diffuse scattering in materials has always been a time-consuming affair, with only large strongly scattering samples being possible to study. In the last decade however, significant technical developments (improvements in supermirror technology, and ${ }^{3} \mathrm{He}$ spin-filters are two examples) have resulted is major increases in efficiency -up to a factor of 60 in the case of D7 compared with the same instrument in the year 2000 . 


\section{ACKNOWLEDGEMENTS}

JRS would like to thank Bob Cywinski and Brian Rainford for introducing him to the wonderful world of magnetic disorder and diffuse scattering. Thanks must also go the D7 millennium project team. They include Ken Andersen, Werner Graf and Daniel Gorny, who developed and produced the 100s of square metres of supermirrors installed on D7. Also, J-F Barthélémy, Pierre Thomas and Pierre George for technical support. And finally my colleagues, Helmut Schober and Amir Murani for their help and guidance.

\section{References}

[1] Web site: http://www.fz-juelich.de/iff/wns_dns.

[2] Web site: http://www.ansto.gov.au/ansto/bragg/hifar/long.html.

[3] Web site: http://www.ill.fr/YellowBook/D7.

[4] Bauer, G., Seitz, E., Just, W., J. Appl. Cryst. 8162 (1975).

[5] Schärpf, O., Capellmann, H., Phys. Stat. Sol. A135 359 (1993).

[6] Schärpf, O., Physica B 174514 (1991).

[7] ILL Yellow Book 2005, ed. Cicognani, G., Institut Laue-Langevin, 2005.

[8] Rauch, H., Waschkowski W., in Neutron Data Booklet, ed. A-J Dianoux and G Lander, Institut Laue-Langevin, 2002.

[9] see Squires, G. L., Introduction to the Theory of Thermal Neutron Scattering, Dover, 1996 - page 173.

[10] see Squires, G. L., ibid. page 134.

[11] Moon, R. M., Riste, T., and Koehler, W. C., Phys. Rev. 181920 (1969).

[12] Sears, V. F., Thermal-Neutron Scattering Lengths and Cross Sections For Condensed-Matter Research, Atomic Energy of Canada, Ltd. Report AECL-8490 (1984).

[13] Jones, T. J. L., and Williams, W. G., Rutherford-Appleton Lab. report RL-77-O79/A (1977).

[14] Abrahams, K., Steinsvoll, O., Bongaarts, P. J. M., and De Lange, P. W., Rev. Sci. Instrum. 33524 (1962).

[15] see Squires, G. L., ibid. page 179.

[16] Ziebeck, K. R. A., and Brown, P. J., J. Phys. F: Metal Physics 102015 (1980).

[17] Wilkinson, C., Räppling and Ziebeck, K. R. A., J. Mag. Magn. Mater. 78269 (1989).

[18] Schärpf, O., and Capellmann, H., Z. Phys. B - Condens. Matter 80253 (1990).

[19] Wildes, A. R., Stewart, J. R., Cowlam, N., Al-Heniti, S., Kiss L. F., and Kemény, T.,J. Phys.: Condens. Matter 15675 (2003).

[20] Mezei, F., and Murani, A. P., J. Mag. Magn. Mater. 14211 (1979).

[21] Ehlers, G., private communication (2005).

[22] Cowley, J. M., Phys. Rev. 7724 (1950).

[23] Warren, B. E., Averbach, B. L., and Roberts, B. W., J. Appl. Phys. 221493 (1951).

[24] Stewart, J. R., and Cywinski, R., J. Mag. Magn. Mater. 272676 (2004).

[25] Kachaturyan, A. G., Theory of Structural Transformations in Solids, Wiley, 1983, page 39.

[26] Cable, J. W., Werner, S. A., Flecher, G. P., and Wakabayashi, N., Phys. Rev. B 291268 (1984).

[27] Tölle, A., Rep. Progr. Phys. 641473 (2001).

[28] Alvarez, F., Colmenero, J., Zorn, R., Willner, L., and Richter, D., Macromolecules 36238 (2003).

[29] Garcia-Hernandez, M., Mompean, F. J., Schärpf, O., Andersen, K. H., Fåk, B., Phys. Rev. B 59 958 (1999). 
[30] Rainford, B. D., J. de Physique, 43 C7-33 (1982).

[31] Marshall, W., J. Phys. C: Solid State Phys., 188 (1968).

[32] An excellent review of myriad chromium-based alloys including $\mathrm{Cr}(\mathrm{Fe})$ is given in: Fawcett, E., Alberts, H. L., Galkin, V. Yu., Noakes D. R., and Yakhmi, J. V., Rev. Mode. Phys. 6625 (1994).

[33] Cywinski, R., and Hicks, T. J., J. Phys. F: Met. Phys. 10693 (1980).

[34] Murani, A. P., J. Mag. Magn. Mater. 22271 (1981).

[35] Cywinski, R., Kilcoyne, S. H., Cox, S. F. J., Scott, C. A., and Schärpf, O., Hyperfine Interact. 64 427 (1990).

[36] Rainford, B. D., Cywinski, R., and Dakin, S. J., J. Mag. Magn. Mater. $140 Đ 144805$ (1995).

[37] Shull, C. G., and Smart, J. S., Phys. Rev. 761256 (1949).

[38] Shull, C. G., Strauser W. A., and Wollan, E. O., Phys. Rev. 83333 (1951).

[39] Hohlwein, D., Hoffmann J. U., and Schneider, R., Phys. Rev. B 68140408 (2003).

[40] D7 has been upgraded as part of the ILL "Millennium Programme". See ILL Annual Report 2003, eds. C. Vettier and G. Cicognani, Institut Laue-Langevin, 2005.

[41] Palmer, S. E., and Chalker, J. T., Phys. Rev. B 62488 (2000).

[42] Rossat-Mignod, J., Neutron scattering Methods in Experimental Physics vol 23C, ed K Skjold and D L Price (New York: Academic) 1987 - page 69.

[43] Champion, J. D. M., Wills, A. S., Fennell, T., Bramwell, S. T., Gardner, J. S., and Green, M. A., Phys. Rev. B 64 140407(R) (2001).

[44] Stewart, J. R., Ehlers, G., Wills, A. S., Bramwell, S. T., and Gardner, J. S., J. Phys.: Condens. Matter 16 L321 (2004).

[45] Nakamura, H., Yoshimoto, K., Shiga, M., Nishi, M. and Kakurai, K., J. Phys.: Condens. Matter 9 4701 (1997).

[46] Stewart, J. R., Rainford, B. D., Eccleston, R. S., and Cywinski, R., Phys. Rev. Lett. 89186403 (2002).

[47] Stewart,J. R., and Cywinski, R., Phys. Rev. B 594305 (1999).

[48] Miyakawa, M., Umetsu, R. Y., Ohta, M., Fujita, A., Fukamichi, K., and Hori, T., Phys. Rev. B 72 054420 (2005) 